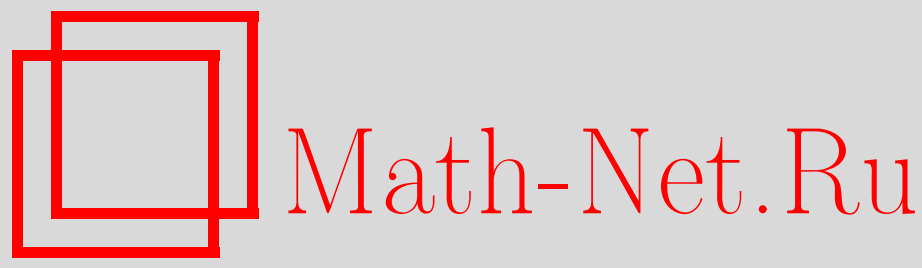

Е. Б. Магадеев, Р. М. Вахитов, Зарождение магнитных неоднородностей на уединенных дефектах ферромагнетика, ТМФ, 2015, том 184, номер 1, 134-144

DOI: https://doi.org/10.4213/tmf8713

Использование Общероссийского математического портала Math-Net.Ru подразумевает, что вы прочитали и согласны с пользовательским соглашением http://www . mathnet.ru/rus/agreement

Параметры загрузки:

IP : 54.198 .55 .26

26 апреля 2023 г., 16:38:21

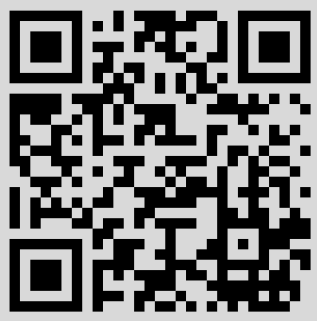




\title{
ЗАРОЖДЕНИЕ МАГНИТНЫХ НЕОДНОРОДНОСТЕЙ НА УЕДИНЕННЫХ ДЕФЕКТАХ ФЕРРОМАГНЕТИКА
}

\begin{abstract}
В рамках микромагнитной теории развит общий подход к задаче по определению условий зарождения магнитных неоднородностей на дефектах одноосного ферромагнетика. Установлено, что при одних и тех же значениях материальных параметров на уединенных дефектах возможно образование двух типов магнитных неоднородностей, соответствующих 0-градусной доменной границе и различающихся амплитудой. Найдены диаграммы их существования и показано, что полученные закономерности зарождения неоднородностей носят общий характер, не зависящий от вида выбранной модели дефекта.
\end{abstract}

Ключевые слова: ферромагнетик, дефект, магнитные неоднородности, уравнение Шредингера, диаграммы существования.

DOI: $10.4213 / \operatorname{tmf} 8713$

\section{1. ВВЕДЕНИЕ}

Реальные магнетики отличаются от их идеализированных моделей прежде всего тем, что в них присутствуют различного рода дефекты, нарушающие их трансляционную инвариантность [1]. Это сказывается на их свойствах и, в частности, влияет на некоторые важные в техническом плане характеристики материала. В то же время интегрирование уравнений Эйлера-Лагранжа [2], описывающих поле вектора намагниченности $\mathbf{M}$ в неоднородной среде, представляется проблематичным [3]. В особенности это касается случая, когда размеры дефектов $L$ и взаимодействующих с ними магнитных неоднородностей $\Delta$ являются величинами одного порядка. Следует отметить, что в определенных ситуациях дефекты могут представлять "потенциальную яму", на которой, как на связанном состоянии, возможно зарождение магнитных неоднородностей, играющих важную роль во многих процессах [4]. Поэтому проблема определения условий возникновения и поведения подобных структур является актуальной. Несмотря на то что для некоторых частных случаев эта задача уже решалась ранее [5]-[10], общего подхода к ней сформулировано не было.

* Башкирский государственный университет, Уфа, Россия.

E-mail: vakhitovrm@yahoo.com 
Кроме того, существующие модели обладают крайне узкой областью применимости и потому их невозможно использовать для количественного описания процессов перемагничивания. В настоящей работе предпринимается попытка ликвидировать эти недостатки путем построения последовательной теории зарождения магнитных неоднородностей на дефектах.

\section{2. ОСНОВНЫЕ ПОЛОЖЕНИЯ}

Рассмотрим ферромагнетик, неоднородный вдоль оси $O y$, и будем предполагать, что все материальные параметры также зависят от координаты $y$. Возникающие в нем магнитные структуры являются блоховскими $(\operatorname{div} \mathbf{M}=0)$ [11] и могут быть описаны единственной функцией $\theta(y)$, характеризующей зависимость угла $\theta$ между вектором $\mathbf{M}$ и осью $O z$ (направленной вдоль оси симметрии) от координаты $y$. Энергию образца, отнесенную к площади сечения плоскостью $x O z$, возьмем в виде следующего функционала:

$$
E=\int_{\mathbb{R}}\left[A(y)\left(\theta^{\prime}(y)\right)^{2}+F(\theta, y)\right] d y,
$$

где $A(y)$ имеет смысл обменного параметра, а функция $F(\theta, y)$ характеризует анизотропные свойства изучаемой системы. Поскольку для ферромагнетика $A(y)>0$, то можно произвести замену $d y \rightarrow d \tilde{y}=d y / A(y)$, и выражение (1) сведется к виду

$$
E=\int_{\mathbb{R}}\left[\left(\theta^{\prime}(\tilde{y})\right)^{2}+\widetilde{F}(\theta, \tilde{y})\right] d \tilde{y}
$$

где $\widetilde{F}(\theta, \tilde{y})=A(y) F(\theta, y)$. Формулу (2) мы принимаем за основу, опуская знак “ ”.

Согласно основным положениям микромагнитной теории [12] уравнение, описывающее распределение намагниченности в образце, имеет вид

$$
\theta^{\prime \prime}=\frac{1}{2} \frac{\partial F}{\partial \theta} .
$$

Будем считать, что функция $F(\theta, y)$ является четной по аргументу $y$ и не зависит от него явно при $|y|>L / 2$, где $L$ имеет смысл ширины дефекта. Тогда локализованной на дефекте магнитной неоднородности отвечает нетривиальное четное решение уравнения (3), стремящееся при $y \rightarrow \pm \infty$ к некоторой постоянной величине $\theta_{ \pm}$. При $y>L / 2$ имеет место равенство $\theta(y)=\theta_{1}\left(y-y_{0}\right)$, где $y_{0}$ - некоторая постоянная величина, а $\theta_{1}$ - нетривиальное решение автономного уравнения, в которое переходит уравнение $(3)$, причем $\theta_{1}(y \rightarrow+\infty)=\theta_{+}$. Ясно, что при $y \rightarrow-\infty$ функция $\theta_{1}(y) \rightarrow \theta_{-}$, причем $\theta_{-}$и $\theta_{+}$можно считать эквивалентными точками минимума функции $F(\theta,|y|>L / 2)$. Таким образом, для асимптотического поведения $\theta_{1}(y)$ имеем

$$
\theta_{1}(y)-\theta_{ \pm} \sim e^{\mp \sqrt{K_{1}} y} \quad \text { при } \quad y \rightarrow \pm \infty,
$$

где

$$
K_{1}=K\left(|y|>\frac{L}{2}\right)>0, \quad K(y) \equiv \frac{1}{2} \frac{\partial^{2} F}{\partial \theta^{2}}\left(\theta_{ \pm}, y\right) .
$$

Ясно, что не для всех рассматриваемых функций $F(\theta, y)$ величина $y_{0}$ при данном выборе $\theta_{1}(y)$ может быть найдена. Если $F(\theta, y)$ непрерывно зависит от некоторого 
параметра $\lambda$, то его значение, при переходе через которое существование магнитной неоднородности становится возможным, определяется условием $y_{0} \rightarrow-\infty$ или $y_{0} \rightarrow+\infty$. Зарождающиеся при этом структуры отличаются амплитудой: в первом случае, о котором мы будем говорить как о 0-градусной доменной границе первого типа ( $0^{\circ}$-ДГ1), она может быть сколь угодно мала, в то время как во втором случае $\left(0^{\circ}\right.$-ДГ2) амплитуда по крайней мере превышает величину $\left|\theta_{+}-\theta_{-}\right|$. Будем считать, что величина $y_{0}$ велика, но конечна. Тогда при $|y| \ll\left|y_{0}\right|$ значения $\theta(y)$ мало отличаются от $\theta_{ \pm}$(здесь и далее верхний знак относится к $0^{\circ}$-ДГ1), и для уравнения (3) относительно $\eta=\theta-\theta_{ \pm}$находим

$$
\eta^{\prime \prime}=K(y) \eta
$$

Полученное уравнение при $y>L / 2$ имеет общее решение

$$
\eta(y)=A_{-} e^{-\sqrt{K_{1}} y}+A_{+} e^{\sqrt{K_{1}} y} .
$$

Учитывая, что $\eta(|y|>L / 2)=\theta_{1}\left(y-y_{0}\right)-\theta_{ \pm}$, и сравнивая (6) и (4), приходим к выводу, что $A_{ \pm}=0$. Таким образом, зарождающейся $0^{\circ}$-ДГ1 соответствует функция $K(y)$, для которой уравнение (5) имеет четное ограниченное решение, исчезающее на бесконечности, что фактически сводит задачу к нахождению собственных значений соответствующего оператора. В то же время в случае $0^{\circ}$-ДГ2 решение, напротив, должно быть “строго неограниченным", т. е. не должно содержать убывающего слагаемого. Следует отметить, что в действительности обе ситуации имеют равные права на существование.

Рассмотрим функцию $\tau(y)=\eta^{\prime}(y) / \eta(y)$. Она является нечетной (в частности, $\tau(0)=0)$, а также удовлетворяет уравнению

$$
\tau^{\prime}+\tau^{2}=K(y)
$$

Следует, однако, иметь в виду, что задача Коши для уравнения (7) не определяет функцию $\tau(y)$, поскольку она сингулярна в нулях $\eta(y)$. Далее заметим, что согласно (6) условие зарождения неоднородности имеет вид

$$
\tau\left(y>\frac{L}{2}\right) \equiv \mp \sqrt{K_{1}} .
$$

Следовательно, если функция $K(y)$ соответствует зарождению $0^{\circ}$-ДГ2, то другая функция $\widetilde{K}(y)$, которой отвечает $\tilde{\tau}(y) \equiv-\tau(y)$, будет соответствовать зарождению $0^{\circ}$-ДГ1, и наоборот. Согласно (7) имеем

$$
\widetilde{K}(y)=-\tau^{\prime}+\tau^{2}=-\frac{\eta^{\prime \prime}}{\eta}+2 \frac{\eta^{\prime 2}}{\eta^{2}} .
$$

Тем самым можно определить нелинейный оператор $\hat{L} K=\widetilde{K}$, действие которого на четную функцию $K(y)$ заключается в том, что решение задачи Коши для уравнения (5) с условиями $\eta^{\prime}(0)=0, \eta(0)=1$ (первое из них выражает четность искомой функции, а второе выбрано произвольно) подставляется в (9). Несложно убедиться, что $\widetilde{K}(0)=-K(0), \widetilde{K}(\infty)=K(\infty), \hat{L}^{2}=1$, а также $\hat{L}$ коммутирует со всеми 
операторами $\widehat{M}_{A} K(y) \equiv A^{2} K(A y)$, образующими группу при $A>0$. Кроме того, примечательно, что константа под действием $\hat{L}$ переходит в модифицированный потенциал Пешля-Теллера [13]: $\hat{L} 1=1-2 / \mathrm{ch}^{2} y$. Оператор $\hat{L}$ позволяет связать между собой задачи для $0^{\circ}$-ДГ 1 и $0^{\circ}$-ДГ2, однако данное обстоятельство носит скорее методический характер из-за серьезного неудобства, вызванного нелинейностью оператора.

Выберем некоторую непрерывную четную функцию $Z(y)$, неубывающую при $y>0$, и рассмотрим такие дефекты, что $K(|y|<L / 2) \equiv Z(y)$. В этом случае $L$ можно выбрать в качестве параметра $\lambda$, причем ясно, что при $L=0$ магнитная неоднородность образоваться не может, а увеличение $L$ свыше значения, при котором происходит зарождение $0^{\circ}$-ДГ, не приведет к ее исчезновению. Следовательно, для решения вопроса о существовании магнитной неоднородности оказывается достаточно при каждом значении $K_{1}$ определить наименьшие $L$, соответствующие условиям зарождения $0^{\circ}$-ДГ1 и $0^{\circ}$-ДГ2. При этом должно выполняться условие $Z(0)<0$, в противном случае форма (2) была бы положительно определена относительно малых отклонений $\theta$ в области дефекта, вследствие чего образование неоднородности оказалось бы энергетически невозможным.

Из уравнения (5) видно, что функция $\eta(y)$ является гладкой. Следовательно, функция $\tau(y)$ непрерывна во всех точках, кроме нулей $\eta(y)$, а потому для зарождающихся неоднородностей из (8) имеем

$$
K_{1}=\tau^{2}\left(\frac{L}{2}\right)
$$

причем положительные значения $\tau(L / 2)$ отвечают $0^{\circ}$-ДГ2, а отрицательные - $0^{\circ}$-ДГ1. $\mathrm{B}$ то же время функция $\tau(y)$ может быть явно определена при $|y|<L / 2$ путем решения уравнения (5), где следует положить $K(y)=Z(y)$, с условиями $\eta^{\prime}(0)=0$, $\eta(0)=1$. Таким образом, соотношение (10) можно рассматривать как уравнение относительно $L$, среди корней которого нас интересуют лишь два наименьших, соответствующих разным знакам $\tau(L / 2)$. Решать его, однако, необязательно, поскольку наибольший интерес представляет диаграмма в осях $L$ и $K_{1}$, показывающая области значений этих параметров, при которых возможно существование магнитной неоднородности. Эти области содержат все точки, лежащие правее хотя бы одной из кривых, задаваемых уравнением (10). Таким образом, вид искомой диаграммы полностью определяется несколькими ветвями этих кривых, лежащими наиболее близко к оси $K_{1}$. При этом ясно, что концами ветвей могут быть либо нули (включая начало координат), либо точки экстремума функции $\tau(y)$, либо одного из концов нет вовсе, что соответствует сингулярности $\tau(y)$.

Отдельно рассмотрим ситуацию $K_{1}=Z(L / 2)$, когда функция $K(y)$ оказывается непрерывной (далее будем называть непрерывным и соответствующий ей дефект). Это может иметь место, например, при локальном нагреве образца лазером [14]. В этом случае параметры $L$ и $K_{1}$ уже не являются независимыми, поэтому введенная здесь диаграмма теряет прежний смысл. В то же время точки $\left(L, K_{1}\right)$, для которых возможно существование магнитных неоднородностей, по-прежнему должны принадлежать областям этой диаграммы, поэтому $L \geqslant L_{0}$, где $L_{0}$ удовлетворяет 
уравнению (10). Тогда из (7) получаем

$$
\tau^{\prime}\left(\frac{L_{0}}{2}\right)=Z\left(\frac{L_{0}}{2}\right)-\tau\left(\frac{L_{0}}{2}\right)^{2}=Z\left(\frac{L_{0}}{2}\right)-Z\left(\frac{L}{2}\right) \leqslant 0,
$$

причем равенство возможно лишь при $L=L_{0}$ и означает пересечение кривой $K_{1}=$ $Z(L / 2)$ с границей области диаграммы в конце ограничивающей ее ветви. Следовательно, значения $K_{1}$, при которых в области дефекта может локализоваться магнитная неоднородность, лежат на ветвях уже построенной нами диаграммы, на которых $\tau(y)$ убывает. Это обстоятельство позволяет полностью исключить необходимость дополнительного анализа случая непрерывного дефекта, который в отрыве от развитого подхода мог бы оказаться весьма затруднительным.

\section{3. ПЛАСТИНЧАТОЕ МАГНИТНОЕ ВКЛЮЧЕНИЕ}

Пусть $Z(y) \equiv K_{2}<0$, что соответствует скачкообразному изменению значений материальных параметров магнетика на границах дефекта при их постоянстве в области дефекта (модель пластинчатого магнитного включения [9], [14]). Решение уравнения (5) имеет вид $\eta=\cos \sqrt{\left|K_{2}\right|} y$, откуда получаем $\tau=-\sqrt{\left|K_{2}\right|} \operatorname{tg} \sqrt{\left|K_{2}\right|} y$. Тогда из (10) для зарождающихся ДГ имеем

$$
\kappa=\operatorname{tg}^{2} \frac{\lambda}{2}
$$

где $\kappa=K_{1} /\left|K_{2}\right|, \lambda=\sqrt{\left|K_{2}\right|} L$. Оставляя на графике этой зависимости две самые левые ветви, отвечающие разным знакам $\tau(L / 2)$, получаем искомую диаграмму (рис. 1). Заметим, что обе ветви имеют вертикальную асимптоту при $\lambda=\pi$, а ветвь, отвечающая $0^{\circ}$-ДГ $2,-$ нуль при $\lambda=2 \pi$. Это означает, что независимо от значения $\kappa$ $0^{\circ}$-ДГ1 может существовать при $\lambda>\pi$, а $0^{\circ}$-ДГ 2 - при $\lambda>2 \pi$. Из (11) также может быть получен явный вид условий, налагаемых на $\lambda$ в зависимости от величины $\kappa$, а именно $\lambda>\pi \mp 2 \operatorname{arctg} \sqrt{\kappa}$.

\section{4. КВАДРАТИЧНАЯ ЗАВИСИМОСТЬ}

Если функция $Z(y)$ гладкая, то в окрестности точки $y=0$ она может быть аппроксимирована квадратичной зависимостью, поэтому рассмотрим случай $Z(y) \equiv$ $K_{2}+\alpha^{2} y^{2}, K_{2}<0, \alpha>0$. Вводя обозначение

$$
G_{i}(x) \equiv G\left(i+\frac{1}{4}\left(1-\kappa_{2}\right), i+\frac{1}{2}, x\right), \quad i=0,1,
$$

где $\kappa_{2}=\left|K_{2}\right| / \alpha$, а $G$ - вырожденная гипергеометрическая функция первого рода [15], из (5) получаем $\eta=e^{-\alpha y^{2} / 2} G_{0}\left(\alpha y^{2}\right)$. Отсюда имеем

$$
\tau=\alpha y\left(-1+\left(1-\kappa_{2}\right) \frac{G_{1}\left(\alpha y^{2}\right)}{G_{0}\left(\alpha y^{2}\right)}\right),
$$

и условие (10) принимает вид

$$
\kappa_{1}=\lambda\left(-1+\left(1-\kappa_{2}\right) \frac{G_{1}(\lambda)}{G_{0}(\lambda)}\right)^{2},
$$

где $\kappa_{1}=K_{1} / \alpha, \lambda=\alpha L^{2} / 4$. 


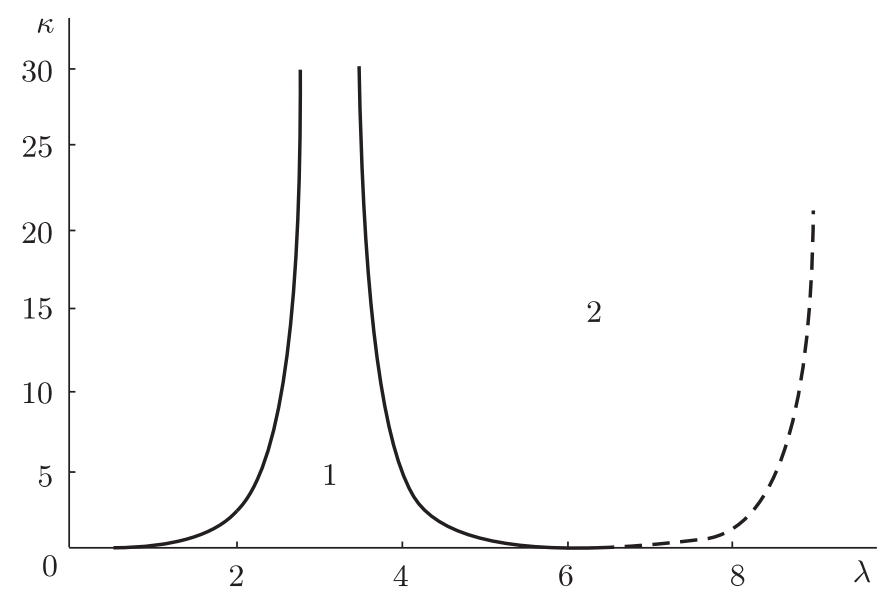

Рис. 1. Области существования магнитных неоднородностей: в области 1 возможно существование только $0^{\circ}$-ДГ1, в области 2 - как $0^{\circ}$-ДГ1, так и $0^{\circ}$-ДГ2. Штриховой линией показана одна из ветвей, не принимаемых во внимание при построении диаграммы.

Поведение функции (12) существенно зависит от значения параметра $\kappa_{2}$. При $\kappa_{2}<\kappa_{0}=1$ она непрерывна и имеет минимум при $\alpha y^{2}=x_{\mathrm{m}}\left(\kappa_{2}\right)$ и нуль при $\alpha y^{2}=$ $x_{0}\left(\kappa_{2}\right)$, где функции $x_{\mathrm{m}}\left(\kappa_{2}\right)$ и $x_{0}\left(\kappa_{2}\right)$ определяются из уравнений

$$
\begin{gathered}
\left(1-\kappa_{2}\right) G_{1}\left(x_{\mathrm{m}}\right)=G_{0}\left(x_{\mathrm{m}}\right)\left(1 \pm \sqrt{1-\frac{\kappa_{2}}{x_{\mathrm{m}}}}\right), \\
\left(1-\kappa_{2}\right) G_{1}\left(x_{0}\right)=G_{0}\left(x_{0}\right) .
\end{gathered}
$$

В этом случае $0^{\circ}$-ДГ1 не может образоваться при значениях $\kappa_{1}$, которые превышают величину, определяемую из выражения (13) при $\lambda=x_{\mathrm{m}}\left(\kappa_{2}\right)$, т. е. с учетом (14) имеем необходимое условие существования $0^{\circ}$-ДГ1 в виде

$$
\kappa_{1}<x_{\mathrm{m}}\left(\kappa_{2}\right)-\kappa_{2} .
$$

Если $\lambda>x_{\mathrm{m}}\left(\kappa_{2}\right)$, то образование $0^{\circ}$-ДГ1 становится возможным независимо от конкретного значения $\kappa_{1}$. Неоднородности типа $0^{\circ}$-ДГ2 могут существовать при произвольных $\kappa_{1}$, однако при этом ограниченной снизу оказывается ширина дефекта, а именно должно выполняться неравенство $\lambda>x_{0}\left(\kappa_{2}\right)$. Таким образом, диаграмма существования $0^{\circ}$-ДГ разных типов в зависимости от значений $\kappa_{1}$ в основном аналогична диаграмме, представленной на рис. 1. В то же время в ней имеется особенность, связанная с наличием области, в которой возможно образование $0^{\circ}$-ДГ 2 , но не $0^{\circ}$-ДГ1 (рис. 2).

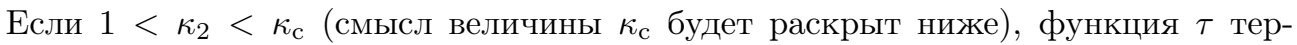
пит разрыв при $\alpha y^{2}=x_{\infty}\left(\kappa_{2}\right)$. Отсюда значения функции $x_{\infty}\left(\kappa_{2}\right)$ определяются наименьшим корнем уравнения

$$
G_{0}\left(x_{\infty}\right)=0
$$




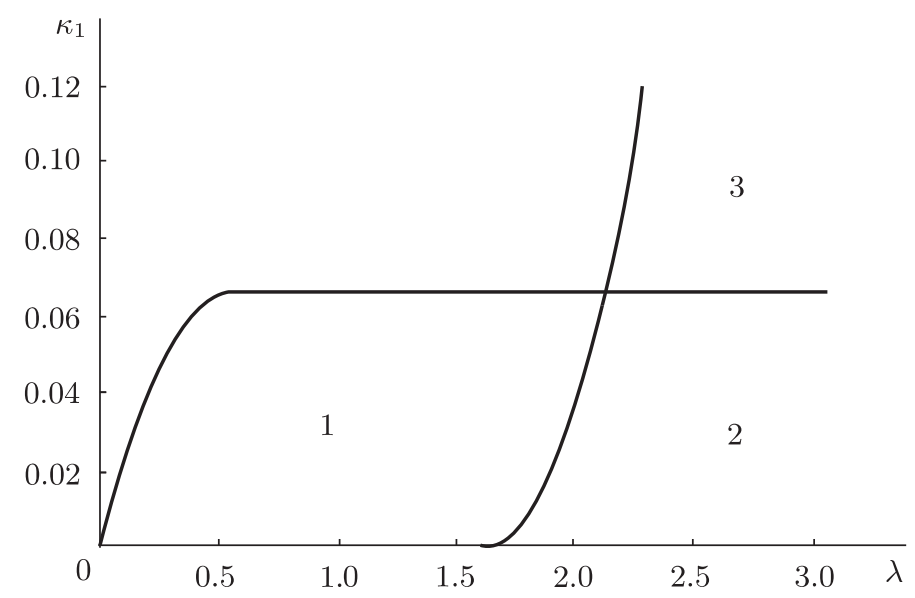

Рис. 2. Диаграмма существования $0^{\circ}$ ДГ обоих типов при $\kappa_{2}=0.5$ : в области 1 возможно существование только $0^{\circ}$-ДГ1, в области 2 - как $0^{\circ}$-ДГ1, так и $0^{\circ}$-ДГ 2 , в области 3 - только $0^{\circ}$-ДГ 2 .

Кроме того, функция $\tau$ имеет минимум при $\alpha y^{2}=x_{\mathrm{m}}\left(\kappa_{2}\right)$. В этом случае $0^{\circ}$-ДГ1 может образоваться при любом значении $\kappa_{1}$, причем достаточным условием является $\lambda>x_{\infty}\left(\kappa_{2}\right)$. В то же время существование $0^{\circ}$-ДГ2 становится невозможным при выполнении условия (16), так что допустимые значения $\kappa_{1}$ оказываются ограничены снизу. При этом условие $\lambda>x_{\infty}\left(\kappa_{2}\right)$ является необходимым, а $\lambda>x_{\mathrm{m}}\left(\kappa_{2}\right)-$ достаточным для образования $0^{\circ}-$ ДГ2.

По мере увеличения $\kappa_{2}$ минимум функции $\tau$ смещается вниз, достигая значения $\tau=0$ при $\kappa_{2}=\kappa_{\mathrm{c}}$. Эта ситуация характеризуется тем, что $x_{\mathrm{m}}\left(\kappa_{\mathrm{c}}\right)=x_{0}\left(\kappa_{\mathrm{c}}\right)=$ $x_{\mathrm{c}}$. Решая совместно уравнения (14) и (15), находим, что $x_{\mathrm{c}}=\kappa_{\mathrm{c}} \approx 4.2872$. При $\kappa_{2}>\kappa_{\text {с }}$ функция $\tau$ имеет нуль при $\alpha y^{2}=x_{0}\left(\kappa_{2}\right)$, поэтому как $0^{\circ}$-ДГ1, так и $0^{\circ}$-ДГ2 могут существовать при произвольных значениях $\kappa_{1}$. Как и в предыдущем случае, условие $\lambda>x_{\infty}\left(\kappa_{2}\right)$ является достаточным для образования $0^{\circ}$-ДГ1 и необходимым для образования $0^{\circ}$-ДГ2. Кроме того, $\lambda>x_{0}\left(\kappa_{2}\right)$ представляет собой достаточное условие существования $0^{\circ}-Д Г 2$.

Для непрерывного дефекта, как было выявлено выше, допустимые значения $\kappa_{1}$ при данном $\kappa_{2}$ определяются участками, на которых функция $\tau$ убывает. Следовательно, при $\kappa_{2}<10^{\circ}$-ДГ2 не может образоваться на данном дефекте, в то время как для $0^{\circ}$-ДГ1 необходимо и достаточно выполнения условия (16). При $\kappa_{2}>10^{\circ}$-ДГ1 может образоваться независимо от значения $\kappa_{1}$, однако $0^{\circ}$-ДГ2 при $1<\kappa_{2}<\kappa_{\mathrm{c}}$ не может существовать, если неравенство (16) справедливо. Таким образом, соответствующая диаграмма области существования $0^{\circ}$-ДГ разных типов в зависимости от $\kappa_{1}$ и $\kappa_{2}$ имеет вид, аналогичный диаграмме, приведенной на рис. 1 (величины, отложенные по осям, разумеется, меняют свой смысл), однако вертикальная асимптота наблюдается при $\kappa_{2}=1$, а нуль - при $\kappa_{2}=\kappa_{\mathrm{c}}$. 


\section{5. НЕГЛАДКИЙ ПРОФИЛЬ ДЕФЕКТА}

В качестве модели подобных дефектов с негладким профилем выберем

$$
Z(y) \equiv K_{2}+\alpha^{3}|y|, \quad K_{2}<0, \quad \alpha>0 .
$$

Из уравнения (5) при $y>0$ имеем

$$
\eta=\frac{\sqrt{3}}{2} \Gamma\left(\frac{1}{3}\right) \Gamma\left(\frac{2}{3}\right)\left(b^{\prime}\left(-\kappa_{2}\right) a\left(\alpha y-\kappa_{2}\right)-a^{\prime}\left(-\kappa_{2}\right) b\left(\alpha y-\kappa_{2}\right)\right),
$$

где $\kappa_{2}=\left|K_{2}\right| / \alpha^{2}, \Gamma(x)$ - гамма-функция, а $a(x)$ и $b(x)$ - функции Эйри первого и второго рода соответственно [15]. Тогда

$$
\tau=\alpha \frac{b^{\prime}\left(-\kappa_{2}\right) a^{\prime}\left(\alpha y-\kappa_{2}\right)-a^{\prime}\left(-\kappa_{2}\right) b^{\prime}\left(\alpha y-\kappa_{2}\right)}{b^{\prime}\left(-\kappa_{2}\right) a\left(\alpha y-\kappa_{2}\right)-a^{\prime}\left(-\kappa_{2}\right) b\left(\alpha y-\kappa_{2}\right)} .
$$

Несмотря на то что данная функция существенно отличается по виду от выражения (12), во всех интересующих нас аспектах их поведение оказывается схожим. Здесь также имеются характерные величины $\kappa_{0}$ и $\kappa_{\mathrm{c}}$, однако их значения другие: $\kappa_{0} \approx 1.0188$ и $\kappa_{\mathrm{c}} \approx 2.9487$. Они являются наименьшими положительными корнями уравнений $a^{\prime}\left(-\kappa_{0}\right)=0, a^{\prime}(0) b^{\prime}\left(-\kappa_{\mathrm{c}}\right)=b^{\prime}(0) a^{\prime}\left(-\kappa_{\mathrm{c}}\right)$. Разумеется, соотношения (14), (15) и (17) применительно к характерным значениям $\alpha y$ для функции (18) также меняются:

$$
\begin{gathered}
b^{\prime}\left(-\kappa_{2}\right) a^{\prime}\left(x_{\mathrm{m}}-\kappa_{2}\right)-a^{\prime}\left(-\kappa_{2}\right) b^{\prime}\left(x_{\mathrm{m}}-\kappa_{2}\right)= \\
= \pm \sqrt{x_{\mathrm{m}}-\kappa_{2}}\left(b^{\prime}\left(-\kappa_{2}\right) a\left(x_{\mathrm{m}}-\kappa_{2}\right)-a^{\prime}\left(-\kappa_{2}\right) b\left(x_{\mathrm{m}}-\kappa_{2}\right)\right), \\
b^{\prime}\left(-\kappa_{2}\right) a^{\prime}\left(x_{0}-\kappa_{2}\right)=a^{\prime}\left(-\kappa_{2}\right) \times b^{\prime}\left(x_{0}-\kappa_{2}\right), \\
b^{\prime}\left(-\kappa_{2}\right) a\left(x_{\infty}-\kappa_{2}\right)=a^{\prime}\left(-\kappa_{2}\right) b\left(x_{\infty}-\kappa_{2}\right) .
\end{gathered}
$$

Следует отметить, что диаграммы, приведенные на рис. 1, качественно верны и для рассматриваемого случая, если принять $\lambda=\alpha L / 2, \kappa_{1}=K_{1} / \alpha^{2}$. Кроме того, в этих обозначениях сохраняет форму и условие (16), вследствие чего диаграмма существования магнитных неоднородностей на данном дефекте вновь отличается от диаграммы на рис. 1 (в осях $\kappa_{2}$ и $\kappa_{1}$ ) только положением асимптоты $\left(\kappa_{2}=\kappa_{0}\right)$ и нуля $\left(\kappa_{2}=\kappa_{\mathrm{c}}\right)$.

\section{6. ОБСУЖДЕНИЕ ПОЛУЧЕННЫХ РЕЗУЛЬТАТОВ}

Сходство результатов, полученных для случаев дефекта с квадратичной зависимостью $Z(y)$ и дефекта с негладким профилем, а также качественное совпадение условий образования $0^{\circ}$-ДГ на дефекте типа пластинчатого магнитного включения и на непрерывных дефектах обоих видов указывают на универсальность наблюдаемых закономерностей. Покажем, что они могут быть получены из общих соображений без конкретизации вида функции $Z(y)$, которую будем считать неограниченной сверху.

Введем обозначения $K_{2}=Z(0)<0, U(y)=Z(y)-K_{2}$, так что $U(0)=0$. Тогда уравнение (5) принимает следующий вид:

$$
-\eta^{\prime \prime}+U(y) \eta=\left|K_{2}\right| \eta .
$$


Если на функцию $\eta(y)$ наложить условие ограниченности, то уравнение (19) будет представлять собой стационарное уравнение Шредингера для частицы, движущейся в потенциальной яме $U(y)$. Соответствующие значения $\left|K_{2}\right|=E_{n}$ будут определять энергию $n$-го уровня, причем $E_{n}>0$ в силу $U(y) \geqslant 0$.

Пусть для начала $\left|K_{2}\right|<E_{0}$. Тогда решение уравнения (19), удовлетворяющее условиям $\eta^{\prime}(0)=0, \eta(0)=1$, имеет максимум в точке $y=0$, поскольку $\eta^{\prime \prime}(0)=-\left|K_{2}\right|<0$. В то же время оно не является ограниченным и нигде не обращается в нуль, поэтому оказывается минимально в некоторой точке $y=y_{0}>0$. Здесь и далее ограничиваясь рассмотрением полуоси $y \geqslant 0$, заметим, что других экстремумов функция $\eta(y)$ не имеет. Действительно, всякая точка ее перегиба в соответствии с (5) является нулем либо самой $\eta(y)$, либо функции $Z(y)$. Последняя, однако, имеет единственный нуль, поэтому число точек перегиба $\eta(y)$, а вместе с тем и экстремумов при $y>0$, превышает число нулей не более чем на 1. Таким образом, при $y>y_{0}$ функция $\eta(y)$ монотонно возрастает. Соответственно, функция $\tau(y)$ обращается в нуль на концах отрезка $\left[0 ; y_{0}\right]$, принимая в некоторой его точке $y=y_{\mathrm{m}}<y_{0}$ минимальное значение, а при $y>y_{0}$ также возрастает, что и приводит к рассмотренному случаю $k_{2}<k_{0}=1$ для дефекта с квадратичной зависимостью. Заметим, что из уравнения (7) вытекают асимптотические соотношения $\tau(y \rightarrow 0+) \approx K_{2} y$ и $\tau(y \rightarrow \infty) \approx \sqrt{K(y)}$ (выбор знака перед корнем обоснован неограниченностью $\eta$ ), справедливые при любых $\left|K_{2}\right|$.

По мере приближения $\left|K_{2}\right|$ к значению $E_{0}$ точка $y=y_{0}$ смещается к бесконеч-

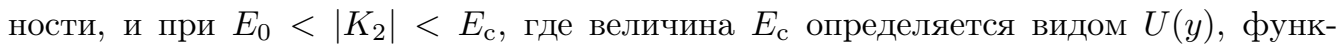
ция $\eta(y)$ оказывается монотонно убывающей и имеет нуль $y=y_{\infty}$. Ясно, что $\tau(y)$ в этой точке обладает сингулярностью, а на луче $y>y_{\infty}$ достигает минимума при некотором $y=y_{\mathrm{m}}$, поскольку при $y \rightarrow \infty$ она должна неограниченно возрастать. Это соответствует случаю $k>k_{\mathrm{c}}=4.2872$. Далее при переходе через $\left|K_{2}\right|=E_{\mathrm{c}}$ у функции $\eta(y)$ появляется дополнительная пара экстремумов, которые совпадают

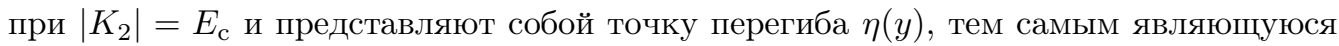
нулем функции $Z(y)$. При дальнейшем увеличении $\left|K_{2}\right|$ минимум $y=y_{0}$ определяет нуль функции $\tau(y)$, а максимум смещается в сторону больших значений $y$, удаляясь на бесконечность при $\left|K_{2}\right|=E_{2}$ (нечетные уровни $E_{n}$ отвечают нечетным собственным функциям $\eta(y)$, а потому не представляют интереса). Следовательно, $E_{\mathrm{c}}<E_{2}$. Дальнейший анализ поведения функции $\eta(y)$ не имеет смысла, поскольку все дополнительные ветви, появляющиеся при больших $\left|K_{2}\right|$, лежат правее уже рассмотренных. Последние, таким образом, имеют вид, показанный на рис. 1, 2, для конкретных $U(y)$ различаясь лишь количественно.

Отдельно следует обсудить условие (16), форма которого, как мы видели, вообще не зависит от $U(y)$. Согласно (10) оно соответствует неравенству $K_{1}<\tau\left(y_{\mathrm{m}}\right)^{2}$, что в силу уравнения (7), а также условия экстремума $\tau^{\prime}\left(y_{\mathrm{m}}\right)=0$ приводит к выражению $K_{1}<Z\left(y_{\mathrm{m}}\right)=-\left|K_{2}\right|+U\left(y_{\mathrm{m}}\right)$, которое с точностью до обозначений совпадает с (16), если в качестве независимой переменной принять не $y$, а $U(y)$, что мы и делали в обоих конкретных случаях. Корректность такой замены переменной продиктована монотонностью и неограниченностью $U(y)$ при $y>0$. Заметим, что условие (16) полностью задает вид диаграммы для непрерывного дефекта, которая в силу универсального характера поведения функции $\tau(y)$ всегда имеет вид, схожий с диаграм- 
мой на рис. 1 , причем положения асимптоты и нуля задаются величинами $E_{0}$ и $E_{\text {c }}$ соответственно. Это, однако, не объясняет справедливости полученных результатов для случая пластинчатого магнитного включения, когда функция $U(y) \equiv 0$ является ограниченной и проведенные нами рассуждения формально неприменимы. В то же время этот случай вполне можно включить в общую схему, если принять для него

$$
U(y)= \begin{cases}0, & |y| \leqslant \frac{L}{2} \\ \infty, & |y|>\frac{L}{2}\end{cases}
$$

Тогда из (19) имеем $E_{n}=(\pi(n+1) / L)^{2}$, откуда для вертикальной асимптоты на рис. 1 получаем $\lambda=\sqrt{E_{0}} L=\pi$, а для нуля $-\lambda=\sqrt{E_{\mathrm{c}}} L<\sqrt{E_{2}} L=3 \pi$, что и было получено ранее. Таким образом, для пластинчатого магнитного включения величина $L$ является характеристикой $U(y)$, а не произвольным внешним параметром, вследствие чего можно говорить о наличии связи, пусть и тривиальной, между $K_{1}$ и $L$. Это приводит нас к парадоксальному выводу, что по своим свойствам модель пластинчатого магнитного включения является моделью непрерывного дефекта, о чем говорят и полученные для нее результаты.

Для случая квадратичной зависимости $U(y)=\alpha^{2} y^{2}$ имеем $E_{n}=\alpha(2 n+1)$, поэтому $\kappa_{0}=E_{0} / \alpha=1, \kappa_{\mathrm{c}}<E_{2} / \alpha=5$. Это означает, что $0^{\circ}$-ДГ1 может образоваться на непрерывном дефекте со сколь угодно большим значением $\kappa_{1}$ лишь в случае $\kappa_{2}>1$. Несмотря на то что данный вывод в рамках построенной теории представляется лишь частным следствием, именно он составляет основу работы [4], позволившей существенно уточнить расчетные значения коэрцитивной силы. Остальные результаты, представленные в указанной работе, относятся к иному выбору профиля потенциальной ямы, что, как видим, не может привести к качественно новым зависимостям. Можно отметить, что в работе [7] также предпринята попытка подобного исследования, построенная на специфическом выборе функции $Z(y)$, что, хоть и приводит к точно интегрируемой модели, представляется все же значительным сужением круга решаемых задач.

\section{7. ЗАКЛЮЧЕНИЕ}

Анализ распределения намагниченности в области уединенного дефекта магнетика однозначно указывает на возможность образования магнитных неоднородностей двух типов, существенно различающихся амплитудой. Вместе с тем, разумеется, должны быть различны и энергии $0^{\circ}$-ДГ1 и $0^{\circ}$-ДГ2, причем интуитивно понятно, что вторые не могут быть энергетически выгоднее первых. Тем не менее при значениях материальных параметров образца вне и внутри дефекта, соответствующих области 3 на рис. 2, $0^{\circ}$-ДГ1 не существует, и $0^{\circ}$-ДГ2 является единственной возможной неоднородностью. Кроме того, эта область допускает дефекты сколь угодно больших размеров, вследствие чего $0^{\circ}$-ДГ2 наверняка может оказаться выгоднее также и однородного состояния. Интересно отметить, что данная ситуация не характерна для модели дефекта с негладким профилем, а вместе с тем - и для пластинчатого магнитного включения, так что исследования, основанные на допущениях этих 
простейших моделей, обречены на неполноту. Кроме того, для последних двух моделей дефектов можно утверждать, что для крупных дефектов, дающих наибольший вклад в технические характеристики материала, однородное распределение намагниченности в их области крайне маловероятно, в то время как $0^{\circ}$-ДГ2, являясь метастабильным состоянием, может дать основной вклад в гистерезисные явления, которые являются доминирующими при перемагничивании изучаемых образцов [1].

Следует отметить, что условия образования магнитных неоднородностей в области уединенных дефектов в конечном итоге определяются не конкретной зависимостью свойств изучаемого образца от пространственных координат, а лишь небольшим числом феноменологических параметров. Это приводит к произволу в выборе модели, на основе которой производится расчет характеристик материала, а потому целесообразно ограничиваться простейшими из них. Так в большинстве случаев разумнее пользоваться моделью пластинчатого магнитного включения, несмотря на некоторые ее недостатки, упоминавшиеся выше.

\section{Список литературы}

[1] D. Goll, "Micromagnetism-microstructure relations and hysteresis loop", Handbook of Magnetism and Advanced Magnetic Materials, eds. H. Kronmüller, S. Parkin, John Wiley \& Sons, New York, 2007, 1023-1058.

[2] H. Kronmüller, "General micromagnetic theory", Handbook of Magnetism and Advanced Magnetic Materials, eds. H. Kronmüller, S. Parkin, John Wiley \& Sons, New York, 2007, 703-741.

[3] Э. Корриган, ТМФ, 171:2 (2012), 271-282.

[4] H. Kronmüller, Phys. Stat. Sol. (b), 144:1 (1987), 385-396.

[5] А. Б. Диченко, В. В. Николаев, ЖЭТФ, 82:4 (1982), 1230-1233.

[6] А. Ф. Кабыченков, В. Г. Шавров, ФТT, 29:1 (1987), 202-203.

[7] М. А. Шамсутдинов, ФТT, 33:11 (1991), 3336-3342.

[8] П. П. Дьячук, Е. В. Лариков, ФТТ, 37:12 (1995), 3735-3737.

[9] Р. М. Вахитов, А. Р. Юмагузин, ФTT, 43:1 (2001), 65-71.

[10] Р. М. Вахитов, Е. Р. Гареева, Е. Б. Магадеев, А. Р. Юмагузин, Вестн. Челлб. гос. ун-та, 9:7 (2011), 22-27.

[11] Е. Б. Магадеев, Р. М. Вахитов, ФTT, 53:5 (2011), 944-950.

[12] З. Флюгге, Задачи по квантовой механике, 1, Мир, М., 1974.

[13] А. С. Логгинов, А. В. Николаев, В.Н.Онищук, П. А. Поляков, Писъма в ЖЭЭТФ, 66:6 (1997), 398-402.

[14] A. Sakuma, J. Magn. Mag. Mater., 88:3 (1990), 369-375.

[15] Н.Н.Лебедев, Специалъные функции и их приложения, Изд-во “Лань", СПб., 2010.

Поступила в редакцию 20.05.2014, после доработки 2.03.2015 